\title{
Duas novas espécies de Megacoelium \\ Szidat, 1954 (Trematoda: Heploporidae), parasitas \\ estomacais de peixes da Amazônia Brasileira, com uma redefiniçāo do gênero
}

\author{
Vernon E. Thatcher ( $\left.{ }^{*}\right)$ \\ Angela B. Varella (")
}

\section{Resumo}

Fol redefinido o gênero Megacoelium Szidat, 1954. (Trematoda: Haploporidae), um parasita do estômago dos bodós (Loricariidae) da América do Sul. Foi indicado que este gênero tem espinhos cuticulares dentro das ventosas e um cirro hermafrodita. As seguintes novas espécies foram descritas no gênero: M. spinispecum $n$. sp. de Pterygoplichthys sp. e M. spinicavum n. sp. de Pterygoplichthys pardalis (Casteinau). As novas espécies distinguem-se do tipo (M. plecostomi Szidat, 1954) por serem maiores e por terem ventosas maiores e um testículo não sinuoso. M. spinispecum é maior que a $\mathbf{M}$. spinicavum, mas tem ventosas e bolsa hermafrodita menores. M. spinispecum também tem um receptáculo seminal uterino, que năo existe na outra espécie.

\section{INTRODUÇÃO}

A família Haploporidae Nicoll, 1914, foi originalmente encontrada nos peixes do litoral europeu. Representantes desta família são conhecidos dos peixes marinhos do gênero Mugil em várias partes do mundo. Algumas espécies são parasitas de peixes de água doce (Martin, 1973). Os Haploporidae dos peixes da América do Sul se conhecem, principalmente, pelos trabalhos de Szidat (1954, 1970, 1971); (Thatcher, 1978); (Thatcher \& Dossman, 1974, 1975) e (Brooks, 1977).

Durante os estudos de ictiopatologia que estão sendo realizados no Instituto Nacional de Pesquisas da Amazônia, Manaus, foram encontrados representantes do gênero Megacoelium Szidat, 1954, nos estômagos de peixes chamados bodó (Siluriformes: Loricariidae). Trata-se de duas novas espécies que são descritas a seguir.

\section{MÉtodos E MATERIAIS}

Os peixes foram capturados pelo pessoal do Departamento de Peixe e Pesca no lago Janauacá, próximo a Manaus, Amazonas, Brasil, e trazidos vivos ao laboratório. Os métodos de necropsiar os hospedeiros e preparar os parasitas estão de acordo com (Thatcher, 1978). Os desenhos foram feitos com a ajuda de uma câmara clara e as medidas com uma ocular micrométrica. As medidas são dos holótipos seguidas pelos limites inferior e superior entre parênteses. Todas as medidas são expressas em milimetros, exceto as dos espinhos cuticulares e dos ovos que são em $\mu \mathrm{m}$.

\section{SEÇÃo SISTEMATICA}

HAPLOPORIDAE Nicoll, 1914

\section{Megacoelium Szidat, 1954}

Dagnose genérica: Corpo alongado, acha. tado lateralmente, estreitando-se poste-iormente. Cutícula com espinhos em fileiras horizontais até à extremidade posterior; com ou sem espinhos dentro das ventosas. Ventosa oral grande, subterminal; prefaringe curta; faringe grande; esôfago curto, envolto com células glandulares; bifurcação antero-dorsal ao acetábulo; cecos medianamente longos, podem ou não ultrapassar o testículo posteriormente; cecos achatados lateralmente, variáveis em largura. Acetábulo grande, pré-equatorial. Testículo único presente, alongado, pós-equatorial, sinuoso ou não. Bolsa hermafrodita piriforme ou ovalada, contendo cirro hermafrodita vesícula seminal interna e células prostáticas. Ve-

(") - Instituto Nacional de Pesquisas da Amazônia, Manaus. 
sícula seminal externa tubular. Poro genital preacetabular, mediano ou submediano. Ovário esférico, mediano, antero-dorsal ao testículo. Útero com alças ventrolaterais de extensão variável; receptáculo seminal uterino presente ou ausente. Ovos numerosos; com ou sem manchas oculares; com pequena projeção polar da casca, na forma de cedilha, oposto ao opérculo.

Parasitas estomacais de peixes da família Loricariidae.

Espécie tipo: M. plecostomi Szidat, 1954.

\section{Megacoelium spinispecum n. sp.}

(Fig. 1 e 2)

Hospedeiro: Pterygoplichthys sp. "Bodó".

Habitat: Estômago.

Procedência: Lago Janauacá, Manaus, Amazonas, Brasil.

Holótipo: Instituto Nacional de Pesquisas da Amazônia (INPA).

Parátipos: INPA e Museu de Zoologia da Universidade de São Paulo.

Diagnose específica (baseada em 5 exemplares): Com as caracteristicas do gênero. Corpo medindo $7,7(5,4-7,7)$ de comprimento e $2, \overrightarrow{8}$ $(2,7-3,0)$ de largura. Cutícula espinhosa até o poro excretor e dentro das ventosas; espinhos cuticulares medem até $20 \mu \mathrm{m}$ de comprimento. Ventosa oral subterminal; mede 0,95 $(0,88-0,95)$ de comprimento e $0,86(0,81-0,88)$ de largura. Faringe esférica, $0,45-0,47$ de diâmetro. Prefaringe curta; esôfago curto. Cecos estendendo-se até à metade do testículo. Acetábulo pré-equatorial; medindo aproximadamente 1,2 de diâmetro. Testículo volumoso, alongado: mede $2,82(1,67-3,25)$ de comprimento e $0,73(0,63-1,0)$ de diâmetro máximo. Bolsa hermafrodita mede $0,84(0,64-1,0)$ de comprimento e $0,45(0,33-0,45)$ de diâmetro máximo. Vesícula seminal externa tubular, sinuosa. Poro genital submediano, entre acetábulo e faringe. Ovário esférico; mede 0,34 $(0,34-0,40)$ de diâmetro. Receptáculo seminal uterino proeminente. Canal de Laurer não observado. Glândulas vitelínicas de folículos com diâmetro variando entre 0,05 e 0,10 . Ovos numerosos, com projeçōes polares em forma de

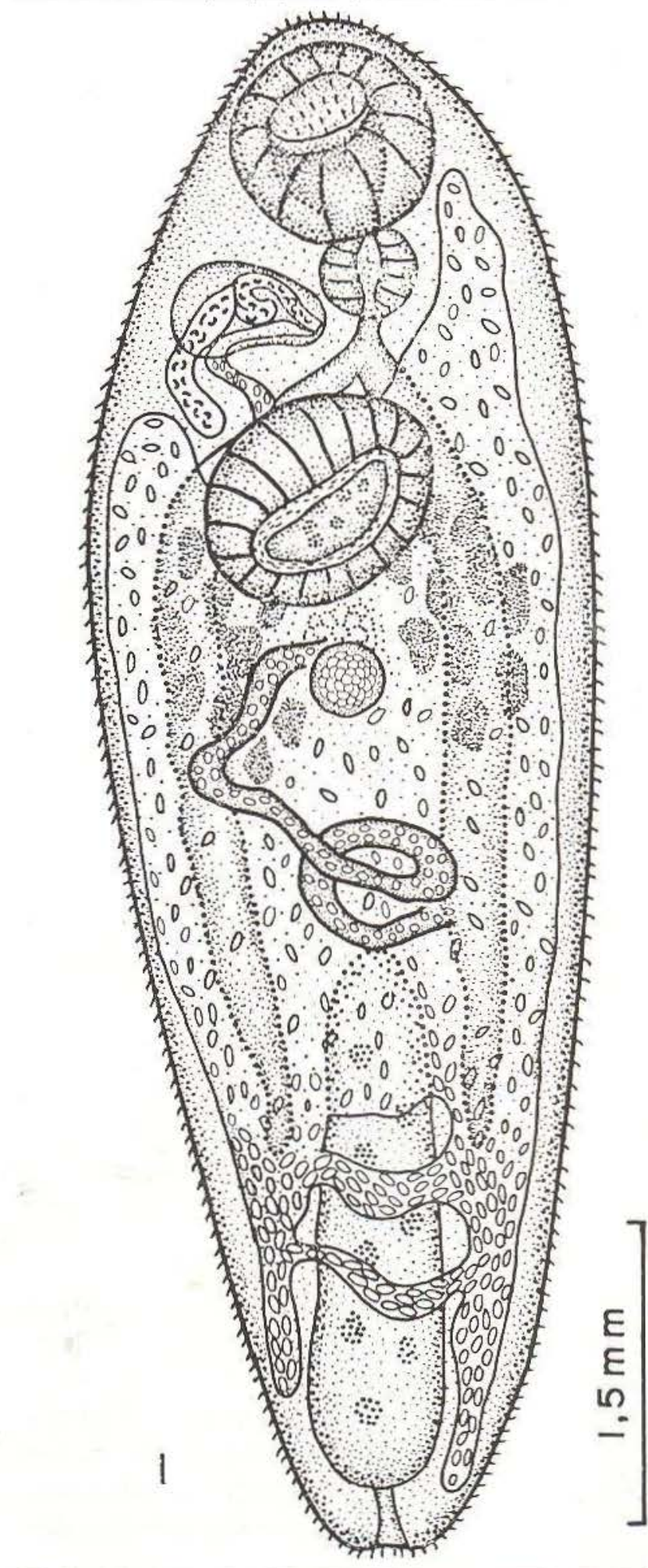

Flg. 1 - Megacoelium spinispecum n. sp. - Vista ventral. 


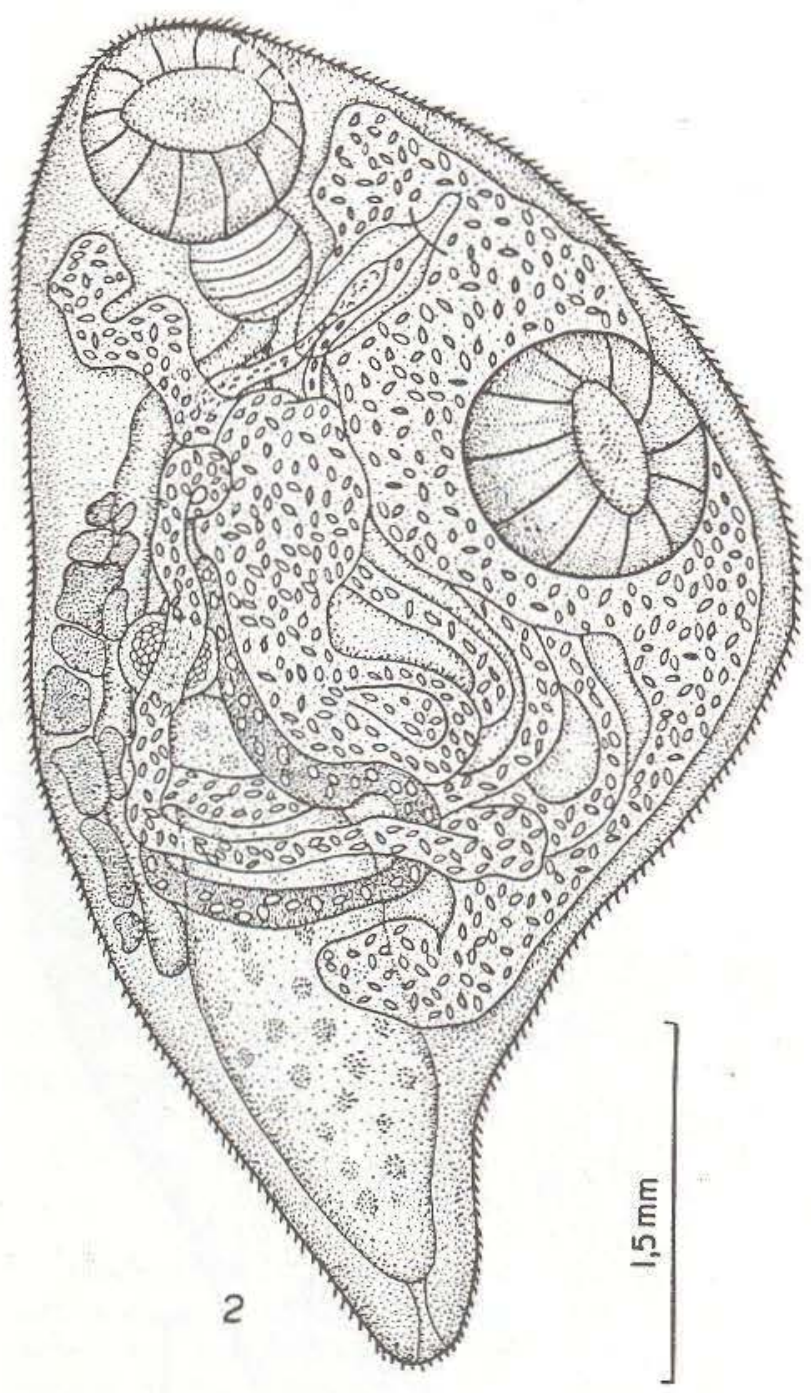

Fig. 2 - Megacoelium spinispecum n. sp. - Vista semilateral.

cedilha e com manchas oculares nos mais ma. duros; medem $43-57 \times 83-100 \mu \mathrm{m}$. Poro excretor terminal.

Megacoelium spinicavum n. sp. (Fig. 3 e 4 )

Hospedeiro: Pterygoplichthys pardalis (Castelnau), "Bodó".

Habitat: Estômago.

Procedência: Lago Janauacá, Manaus, Amazonas, Brasil.

Holótipo: Instituto Nacional de Pesquisas da Amazônia (INPA).

Parátipos: INPA e Museu de Zoologia da Universidade de São Paulo.
Diagnose específica (baseada em 23 exem. plares): Com as características do gênero. Corpo medindo $3,5(3,5-4.9)$ de comprimento e $1,2(1,2-1,7)$ de profundidade. Cutícula espinhosa até o poro excretor e dentro das ventosas, espinhos cuticulares medem até $22 \mu \mathrm{m}$ de comprimento. Ventosa oral subterminal; mede $0,52(0,52-0,58)$ de comprimento e $0.48(0,48-0,55)$ de largura. Faringe esférica, 0,27-0,37 de diâmetro. Prefaringe curta: esôfago curto. Cecos estendendo-se até à me. tade do testículo. Acetábulo pré-equatorial; mede $0,66(0,64-0,75)$ de comprimento e 0,55 $(0,55-0,79)$ de largura. Testículo volumoso, alongado; mede $1,1(1,1-2,45)$ de comprimento e $0,53(0,47-0,73)$ de diâmetro. Bolsa hermafrodita mede $0,45(0,37-0,64)$ de comprimento e $0,21(0.21-0,30)$ de diâmetro. Vesícula seminal externa tubular. Poro genital submediano. entre o acetábulo e a faringe. Ovário esférico; mede 0,21-0,39 de diâmetro. Receptáculo seminal uterino ausente. Canal de Laurer não observado. Glândulas vitelínicas de folículos com diâmetro variando entre 0,04 e 0,09 . Ovos numerosos; com projeçōes polares em forma de cedilha; sem manchas oculares; medem 50-60 $\times$ 95-110 $\mu \mathrm{m}$. Poro excretor terminal.

\section{Discussão}

As duas novas espécies aqui descritas mostram semelhanças com Megacoelium plecostomi Szidat, 1954, de um bodó argentino (Plecostomus plecostomus L.). Esta última é a única espécie conhecida no gênero de acordo com Travassos et al. (1969) e com Yamaguti (1971). Pela forma e distribuição dos órgãos principais e pelo habitat dos vermes, é evidente que as novas espécies pertencem ao gênero de Szidat (1954). As novas formas mostram espinhos dentro das ventosas e um cirro hermafrodita, características não citadas para M. plecostomi, mas é provável que a espécie argentina também tenha estas estruturas. O nome Megacoelium foi escoihido por Szidat porque ele ficou impressionado com a largura dos cecos. Infelizmente, esta característica é muito variável, de acordo com os estudos da presente série. Aliás, como os vermes são late- 
ralmente achatados, os cecos são mais amplos no sentido vertical (vista ventral) que largos, no. sentido lateral.

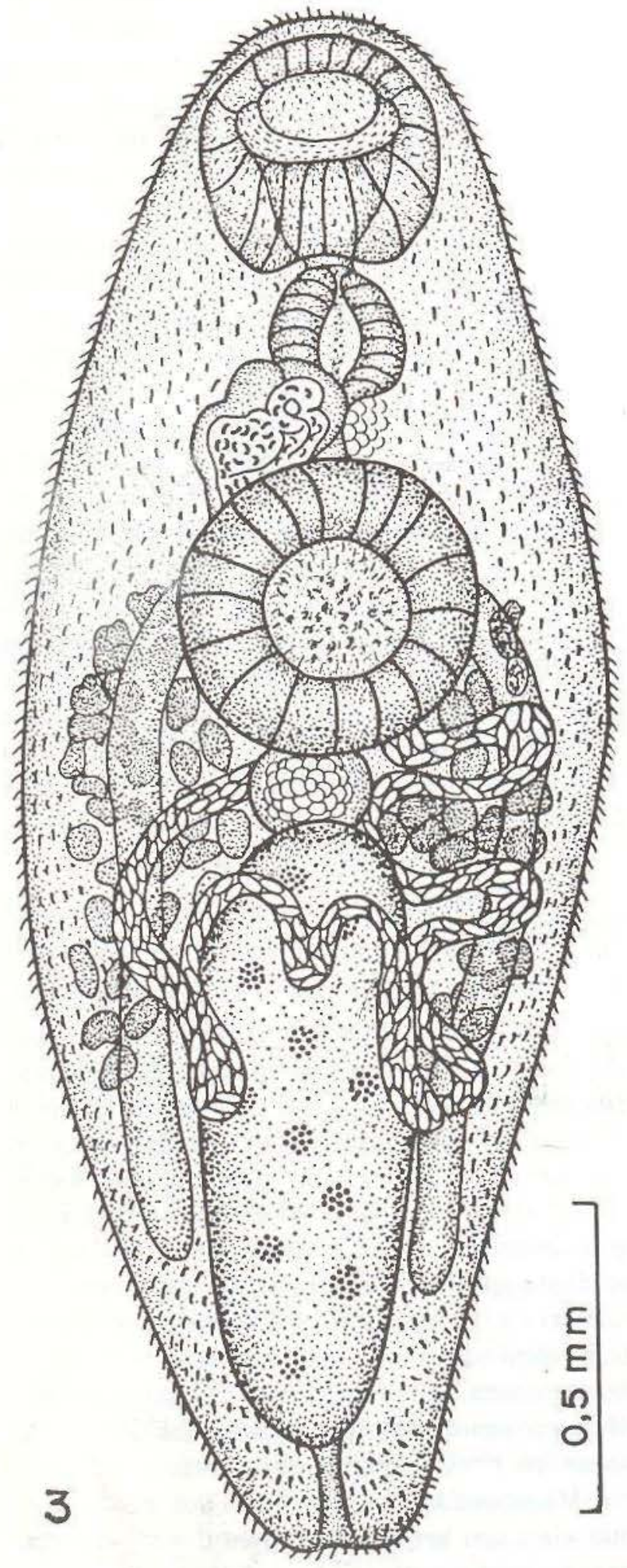

Flg. 3 - Megacoelium spinicavum n. sp. - Vista ventral.

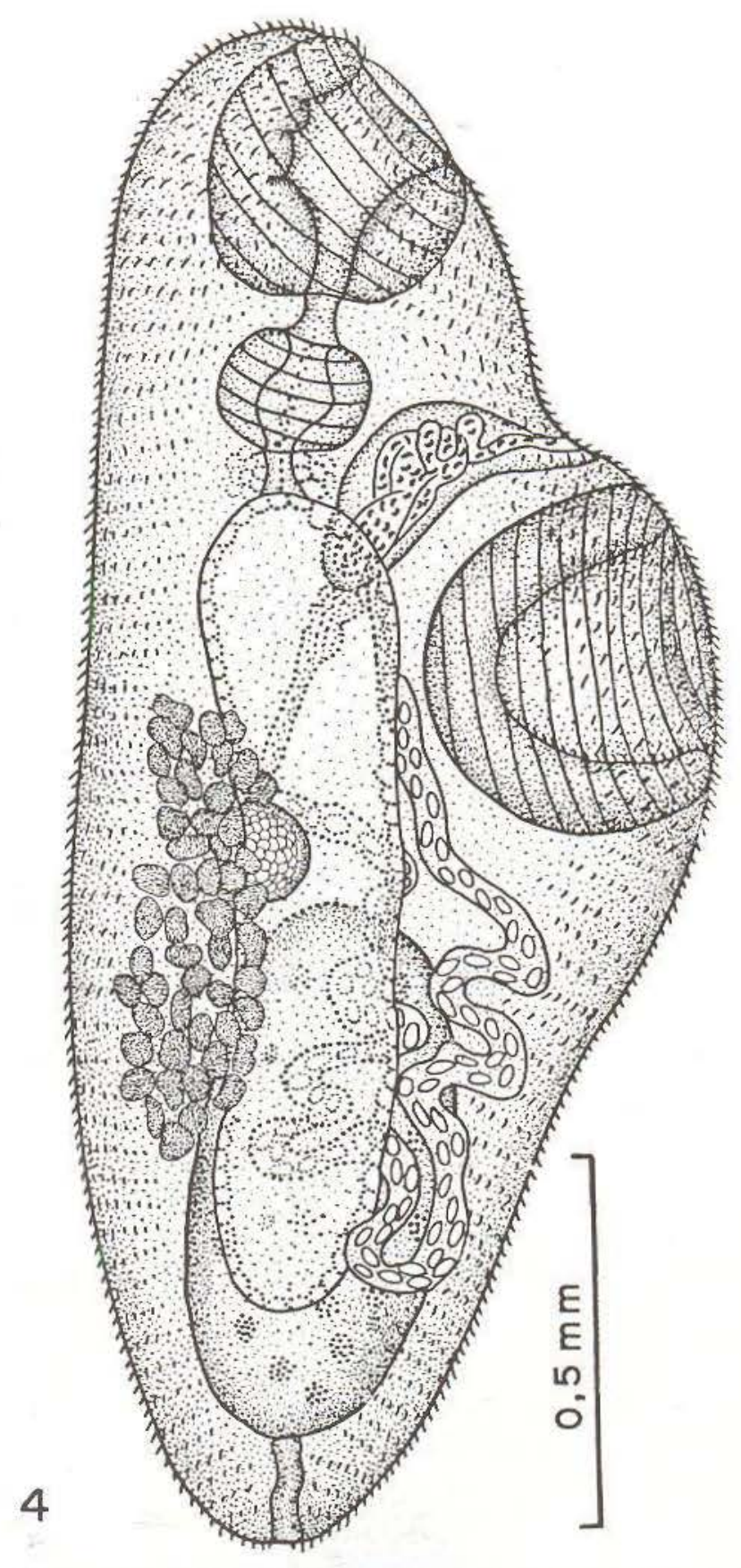

Fig. 4 - Megacoelium spinicavum n. sp. - Vista lateral.

Ambas as novas espécies são notavelmente maiores que $M$. plecostomi e têm ventosas relativamente maiores e testículo mais volumoso. Aliás, o testículo, em nenhuma das duas espécies aqui consideradas é sinuoso, o que foi citado para $M$. plecostomi. As duas novas espécies distinguem-se entre si pelas seguintes características: 1) $M$. spinispecum tem um receptáculo seminal uterino proeminente, 
mas M. spinicavum não tem esta estrutura; 2) $M$. spinispecum tem as ventosas relativamente menores que as de $M$. spinicavum; 3) A bolsa hermafrodita é menor em $M$. spinispecum comparada com a outra espécie; 4) Os cecos de $M$. spinispecum são delgados e na outra espécie são mais amplas; 5) $M$. spinispecum é maior que $M$. spinicavum.

\section{Etrmologia}

Em latim, spinus = espinho e cavus ou specus $=$ caverna ou buraco. Ambos os nomes específicos aqui introduzidos fazem referência aos espinhos dentro das ventosas, uma característica pouco comum entre os trematódeos.

\section{Agradecimentos}

Agradecemos à desenhista, Srta Maria de Fátima Vieira, pela ajuda prestada na elaboração dos desenhos.

\section{SUMMARY}

Megacoelium Szidat, 1954 (Trematoda Haploporidae), a stomach parasite of South American loricariid catfish, was redefined. This genus was found to have cuticular spines within the suckers and a hermaphroditic cirrus. Two new species were described in the genus, namely: M. spinispecum $n$. sp. from Pterygoplichthys $\mathrm{sp}$. and M. spinicavum n. sp. from Pterygoplichthys pardalis (Castelnau). The new species differ from the type (M. plecostomi Szidat, 1954) in being much larger and in having larger suckers and a nonsinuous testis. M. spinispecum is larger than M. spinicavum and has smaller suckers and a smaller hermaphroditic sac. M. spinispecum also has a prominent receptaculum seminis uterinum which the other species lacks.

\section{REFERENNCIAS BIBLIOGRÁFICAS}

BROOKS. D.R.

1977 - A new genus and two new species of trematodes from characid fishes in $\mathrm{Co}$. lombia. Trans. Amer. Mic. Soc., 96(2): 267-270.
MARTIN, W.E.

1973 - Life history of Saccocoelioides pearsoni n. $\mathrm{sp}$, and the description of Lecithobotrys sprendi $n$. sp. (Trematoda: Haploporidae). Trans. Amer. Mic, Soc., $92: 80-95$.

SZIDAT, L.

1954 - Tremátodos nuevos de peces de agua dulce de la República Argentina y un intento para aclarar su carácter marino. Rev. Mus. Nac. Cienc. Nat. "Bernardino Rivadávia", 3 : 1-85.

1970 - Saccocoelioides octavus $n$. sp. una nueva espécie del género Saccocoelioides Szidat 1954 (Trematoda, Haploporinae Looss 1902). Rev. Mus. Argentino Cienc. Nat. "Bernardino Rivadávia", $10: 87-100$.

1971 - Sobre una nueva espécie del género Sacco. coelloides Szidat 1954 de Astyanax bipunctatus, Saccocoelioides bacilliformis $\mathrm{sp}$. nov. del Río Reconquista, Provincia de Buenos Aires. Comun. Mus. Argentino Cienc. Nat, "Bernardino Rivadávia", 1(7): 97-100.

THATCHER, V.E.

1978 - Quatro espécies novas da familia Haplo. poridae (Trematoda; Digenea) de peixes de água doce da Colômbia, com uma re. visão do gênero Saccocoelioides Szidat, 1954. Acta Amazonica, $8: 477-484$.

THATCHER, V.E. \& DOSSMAN, M.D.

1974 - Lecithobotrioides mediacanoensis n. g., n. sp. (Trematoda: Haploporidae) from a freshwater fish (Prochilodus reticulatus) in Colombia. Trans. Amer. Mic. Soc., 93: 261-264.

1975 - Unicoelium prochilodorum gen, et sp. n. (Trematoda: Haploporidae) from a freshwater fish (Prochilodus reticulatus) in Colombia. Proc. Helm. Soc. Wash., $42: 28-30$

TRAVASSOS, L.; TEIXEIRA DE FREITAS, J.F.; KOHN, A. 1969 - Trematódeos do Brasil. Mem. Inst. Oswaldo Cruz, $67: 1.886$.

YAMAGUTI, $\mathrm{S}$.

1971 - Synopsis of Digenetic Trematodes of Vertebrates. Keigaku Publ. Co. Tokio. $1772 \mathrm{p}$.

(Aceito para publicação em 27/11/80) 